
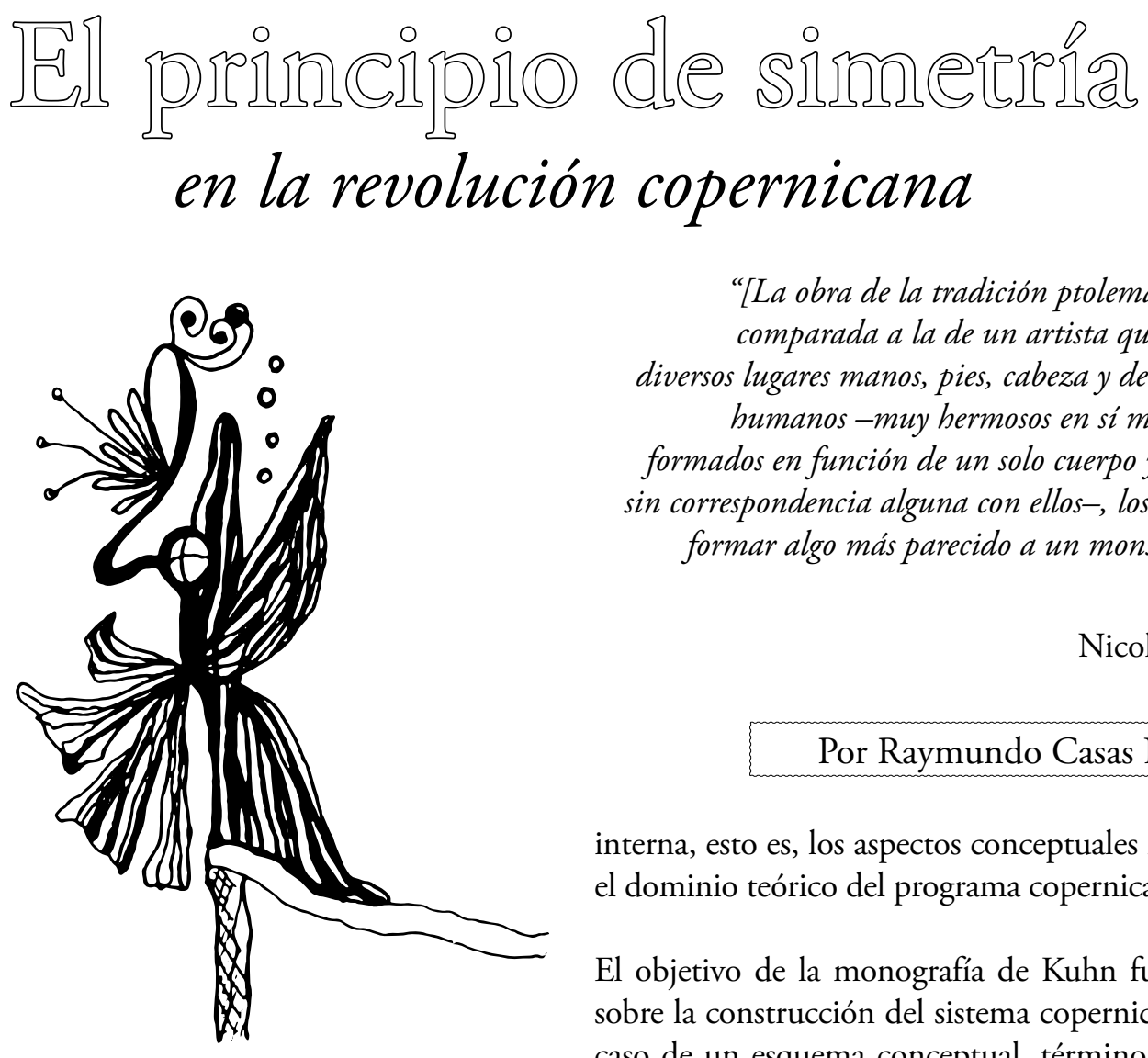

\author{
"[La obra de la tradición ptolemaica] puede ser \\ comparada a la de un artista que, tomando de \\ diversos lugares manos, pies, cabeza y demás miembros \\ humanos-muy hermosos en si mismos, pero no \\ formados en función de un solo cuerpo y, por lo tanto, \\ sin correspondencia alguna con ellos-, los reuniera para \\ formar algo más parecido a un monstruo que a un \\ hombre". \\ Nicolás Copérnico \\ Por Raymundo Casas Navarro
}

interna, esto es, los aspectos conceptuales implicados en el dominio teórico del programa copernicano.

El objetivo de la monografía de Kuhn fue echar luces sobre la construcción del sistema copernicano como un caso de un esquema conceptual, término que contiene en ciernes la noción clásica de paradigma de la nueva

Desde el clásico estudio de Kuhn (1957), mucho se ha insistido en la pluralidad estructural de la llamada revolución copernicana -esto es, la sustitución de la astronomía ptolemaica centrada en la Tierra por la astronomía copernicana centrada casi en el Sol-. El poliedro de la revolución copernicana incluye aspectos matemáticos, astronómicos, físicos, filosóficos y teológicos. Sin duda, la comprensión de la estructura y la dinámica de laciencia no se puede restringir a los aspectos fácticos o positivos como pretendía Comte: hay que considerar lo que Burtt (1959) llama fundamentos metafísicos de la ciencia. Si bien todos estos aspectos merecen especial atención en el caso de la llamada revolución copernicana, son dos perspectivas las más conspicuas: la astronomía matemática con sus constructos y cómputos y la filosofía mística de raigambre pitagóricoplatónica (en la que el Sol deviene el arquetipo de mayor esplendor). De acuerdo con el tratado de Kuhn, un trabajo verdaderamente capital e imponente, el carácter poliédrico de la revolución copernicana se puede mostrar en que implicó el desarrollo de una nueva física, una nueva concepción del espacio y una nueva idea de la relación entre los hombres y la divinidad.Aunque el significado de 'revolución copernicana' debe abarcar todos esos matices, en este ensayo nos circunscribiremos a analizar la historia filosofía de la ciencia (Kuhn, 1971). El estudio kuhniano hace un despliegue analítico impresionante desde el simple modelo del universo de las dos esferas del mundo antiguo hasta el modelo copernicano anclado en la cultura del Renacimiento.

A partir de la dilucidación de las funciones o roles de los esquemas conceptuales en las tradiciones científicas, Kuhn intenta comprender la necesidad de la revolución copernicana. En su mirada, un esquema conceptual (a saber, un preconstructo de la noción de paradigma) incluye, por lo menos, tres componentes: (a) un aparato teórico desarrollado a partir de las observaciones, pero que las trasciende, desde el punto de vista lógico; (b) un criterio de economía conceptual, esto es, el esquema debe constituir un buen epítome del sistema real para facilitar el trabajo de la memoria humana; (c) una visión del mundo, es decir, un sistema de creencias sobre el cosmos, el hombre, la divinidad. Gracias al funcionamiento de los tres componentes, el esquema conceptual permite explicar los fenómenos (operación estrictamente lógica) y nos brinda un marco inteligible del mundo (asunto esencialmente psicológico). Además de ayudar a explicar y comprender los fenómenos, el esquema conceptual nos provee un mecanismo de 
previsión con el cual el científico puede explorar con éxito lo desconocido. Así, el navegante Colón llegó a descubrir un nuevo continente (América), apoyándose en el esquema de Ptolomeo.

En el tratado de 1957 se puede vislumbrar la explicación kuhniana de la irrupción de la revolución copernicana, lo que se consolidará en la obra posterior que presenta la teoría de Kuhn sobre la estructura de las revoluciones científicas. Gracias a un análisis erudito y minucioso, Kuhn tratará de mostrar que el paradigma ptolemaico se encontraba en un estado de crisis, es decir, el paradigma forjado lentamente por Platón, Eudoxo, Aristóteles, Hiparco y, principalmente, Ptolomeo se disgregaba en versiones que quebraban la unidad monolítica paradigmática. Debido a que cada astrónomo estaba en libertad de proponer su propio sistema de círculos, de acuerdo con Kuhn, se llegó a una situación de variación intolerable, el caldo de cultivo para la emergencia de un nuevo paradigma: el copernicano.

Debemos decir que la explicación kuhniana ha sido puesta en tela de juicio por un historiador de la ciencia competente y conocedor de la obra copernicana (véase, por ejemplo: Gingerich, 2004; y Gingerich \&MacLachlan, 2005). En un ensayo histórico revelador (1975), Owen Gingerich sostiene que el estado de crisis aludido por Kuhn no se sustenta en un análisis histórico del momento en que Copérnico formuló su innovación. Según Gingerich, al leer las motivaciones de Copérnico, se puede deducir que el astrónomo polaco no estaba de acuerdo con el sistema de Ptolomeo por consideraciones estéticas y no se puede dar cuenta de la insatisfacción de Copérnico a partir de una hipótesis de un periodo de crisis de paradigma, en términos de Kuhn. Es decir, según la exégesis de Gingerich, la deformidad del sistema ptolemaico nació con el mismo Ptolomeo y no fue una lenta acumulación de anomalías que condujo a un estado de crisis de paradigma, según el típico expediente kuhniano. La importancia de la consideración estética se puede mostrar con el análisis del Commentariolus, el primer opúsculo copernicano, escrito hacia 1510 (E. Rosen, 1959) y con la lectura de la principal obra copernicana: el De revolutionibus de 1543 (Copérnico, 2009). Dado que Copérnico estaba guiado por una visión estética de la estructura del universo, la revolución copernicana se puede entender como el producto de una búsqueda apasionada de simetrías. Probablemente, Copérnico era partícipe del criterio de Dirac, según el cual la belleza de una teoría científica es aun más importante que el peso de las pruebas experimentales. Según Dirac, las leyes físicas deben ser matemáticamente bellas, lo que se ejemplifica claramente en la propia ecuación de Dirac.

Justamente, esta inmersión de Gingerich en el análisis historiográfico nos permite formular una interpretación sobre el estatus epistémico de la innovación copernicana. Se puede, a nuestro juicio, demostrar con claridad -tal es el objetivo del presente ensayo-que las consideraciones estéticas (ligadas fundamentalmente con el principio de simetría) fueron determinantes para que Copérnico tuviera la necesidad de transferir a la Tierra los movimientos atribuidos tradicionalmente al Sol. Si hubo una verdadera revolución copernicana (algo que puede ser objeto de discusión como hemos hecho en Casas y Matta, 2006: pp. 65-77), se trata de una revolución que atañe a una mirada estética sobre la estructura y la arquitectura del universo. La llamada revolución copernicana no dependió crucialmente de consideraciones fácticas, matemáticas o de una crisis kuhniana de paradigma, sino de una intelección coherente sobre la simetría del cosmos, un principio formulado por Platón cuando estaba bajo el influjo de Pitágoras. Copérnico fue el primero en percatarse de que el sistema ideado por Ptolomeo no era acorde con el principio platónico de simetría y ese entendimiento lo condujo a proponer una hipótesis audaz (el movimiento de la Tierra) que eliminaba las aberrantes asimetrías introducidas por Ptolomeo. Para apuntalar mejor nuestra interpretación, delinearemos el trayecto de la tradición antigua de la astronomía y, luego, nos centraremos en el Commentariolus, pequeño tratado en el que Copérnico expone su visión en términos cualitativos, esto es, sin el aparato matemático imponente del De revolutionibus.

Antes de embarcarnos en el crucero de la historia interna de la ciencia, conviene aclarar el uso de la noción de simetría en ciencia. En trabajos como los de Weyl (1952) y J. Rosen (1975), se expone que la noción científica de simetría se da en dos sentidos imbricados. Por un lado, la simetría se entiende como armonía, razón por la cual la búsqueda de simetrías se entiende como una búsqueda de armonías (convenientes proporciones y correspondencias de unas cosas con otras). Por otro lado, la simetría se define como una regularidad geométrica, lo que se traduce en una secuencia estable, repetitiva y ordenada; razón por la cual la simetría es sinónimo de belleza: un mundo simétrico es, por ello, un cosmos bello. Con miras a evaluar una teoría científica, en este sentido, se puede decir que la mejor teoría es la que plasma simetrías o elimina las asimetrías; asimismo, se puede decir que 
una teoría defectiva se caracteriza por alejarse de las simetrías o que anida asimetrías.

La noción de simetría en ciencia es importante en la medida en que nos brinda una teoría de la belleza como una gran concepción, un gran sistema, una profunda visión (Tatarkiewciz, 2001). Además, la simetría tiene un valor heurístico fundamental, según la argumentación desarrollada en un profundo ensayo de Enrique Álvarez Vita (2011). Álvarez Vita sostiene que la belleza motoriza la búsqueda científica y defiende su punto de vista con un análisis iluminador de la gesta de grandes hombres de ciencia como Einstein y Dirac. Así, guiado por el sentido del factor estético, el científico (por ejemplo, Copérnico en la astronomía) va en busca de simetrías. Esa búsqueda llega a su cúspide cuando el hombre de ciencia logra hallar simetrías arcanas, armonías que nadie antes había podido prever. Este análisis bien nos puede dar una nueva definición de ciencia: la búsqueda de las simetrías arcanas del cosmos.

El principio de simetría no es un concepto exclusivo de la física contemporánea. puede retrotraer hasta los tiempos prístinos de la

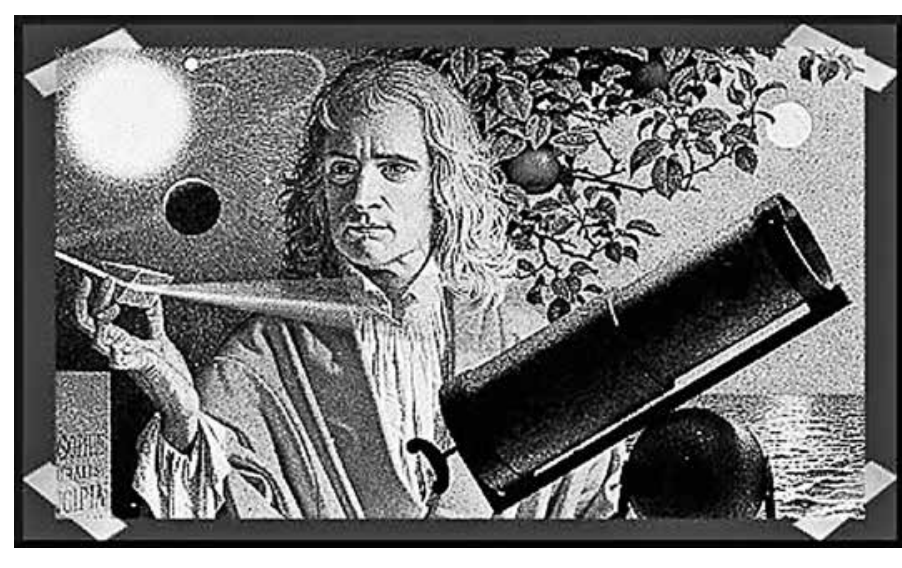

es, la esfera), una órbita perfecta (esto es, circular) y una velocidad perfecta (esto es, constante). Según el axioma platónico propugnado en el Timeo, los astrónomos matemáticos tenían que explicar los movimientos planetarios con un sistema de círculos que exhiba un movimiento simétrico.

Inicialmente, la astronomía antigua formuló un modelo muy simple: el universo de las dos esferas. Luego, con pasos lentos pero seguros, erigió modelos más sofisticados en una verdadera arquitectura del cosmos. En esa construcción tuvo valor gravitante el principio armónico de simetría tal como se expone en el Timeo platónico. En el universo esférico y circular, las estrellas y los planetas exhiben una revolución uniforme y rotación circular en torno a la Tierra, el centro estático del universo. El principio platónico exigía a priori que el sistema global del universo debía caracterizarse como simétrico. El movimiento de las estrellas y del cuerpo solar se explicó coherentemente con el principio de la simetría, lo que cabe entender como un gran logro de la astronomía antigua de raigambre pitagóricoplatónica. Sin embargo, ciencia occidental y, en la astronomía o física celeste de la Antigüedad, tiene una fuente definida: el pensamiento platónico. En el Timeo (un diálogo con clara influencia pitagórica), se formula el principio de simetría aplicado a los movimientos cósmicos (Platón, 1993). En la perspectiva platónica, la simetría se traduce en el carácter armónico de los movimientos de los cuerpos celestes. Las estrellas y los planetas son esferas perfectas (esto es, figuras simétricas) con curvas regulares y con repeticiones rítmicas (esto es, movimientos simétricos). La sinfonía del mundo debía traducirse en la belleza matemática de los modelos del universo. Este principio o axioma señaló un reto decisivo a los astrónomos matemáticos, quienes debían idear sistemas de círculos acordes con la simetría global del cosmos. En el diálogo platónico está el germen del programa científico de la tradición antigua de la astronomía. Al ser un programa de investigación, sentó las bases para una heurística, esto es, una cohorte de rutas de indagación en concordancia con el principio de simetría también conocido como el principio de perfección: Los cuerpos celestes, qua naturalezas perfectas, tienen una forma perfecta (esto los movimientos planetarios constituyeron un desafío acuciante para la astronomía antigua. Si bien el Sol podía ser descrito según el principio de simetría (con la introducción del constructo de eclíptica), el movimiento de los demás planetas (Luna, Mercurio, Venus, Marte, Júpiter y Saturno) se revelaba mucho más complejo. En particular, fue un terrible rompecabezas el movimiento llamado retrógrado que exhibían los planetas. Así, el programa de la astronomía antigua tenía retos graves como ajustar el movimiento de Marte, por poner un caso, al principio de simetría. No era un problema meramente conceptual o de índole filosófica, sino un problema cuantitativo y de cómputo. La astronomía antigua pronto se desarrolló como una ciencia que usaba sistemáticamente el lenguaje matemático y ello nos da la comprensión del nivel de sofisticación al que llegó esta tradición.

En virtud de que los movimientos planetarios se veían como asimétricos, el reto platónico estribaba en postular una serie de movimientos uniformes y regulares que podrían dar cuenta de los movimientos aparentes de 
los planetas. Este reto recibió un nombre técnico en la tradición: salvar las apariencias.

Así, el modelo de las esferas homocéntricas de Eudoxo constituyó un avance importante en el trayecto de la tradición antigua. Se trataba de una solución ingeniosa, matemáticamente impecable, pero que no brindaba un mecanismo adecuado para dar cuenta de los movimientos retrógrados de los planetas. Por ejemplo, el modelo de Eudoxo predecía que el brillo de los planetas debía ser uniforme, lo que no correspondía con las observaciones astronómicas. El fracaso de Eudoxo condujo al modelo de epiciclos y deferentes de Hiparco. El epiciclo es un pequeño círculo que gira uniformemente alrededor de un punto situado sobre la circunferencia de un segundo círculo en rotación (el denominado deferente). El sistema epiciclo-deferente llegó a tal nivel de sofisticación que se plasmó en un torbellino de bucles para explicar sistemáticamente algunas irregularidades observadas en la danza de los planetas.

A pesar de que el incremento de bucles en el modelo de epiciclos y deferentes resolvía algunas asimetrías en el movimiento planetario, también generaba ciertas anomalías recalcitrantes (por ejemplo, la posición real del planeta no concordaba con la posición teórica predicha por el modelo). Se intentaba resolver estas anomalías con nuevos ajustes en el modelo de epiciclos y deferentes, de acuerdo con el programa de investigación de Hiparco.

Esta era la situación cuando entró en escena la genial obra de Ptolomeo: el Almagesto (siglo II de nuestra era).Ptolomeo configuró mejor el sistema de Hiparco y desarrolló una astronomía planetaria cuya misión fundamental era «salvar las apariencias», esto es, resolver el problema de los planetas con el modelo de epiciclodeferente. Una innovación importante, desde el punto de vista heurístico, es la distinción entre epiciclos mayores (un número fijo de cinco) y epiciclos menores (un número variable). Dado que todavía pervivían ciertas asimetrías en el movimiento de los planetas, Ptolomeo introdujo el artilugio técnico del ecuante o punctum aequans. El rasgo distintivo que define al ecuante es que la velocidad de rotación de un deferente viene a ser uniforme no respecto de su centro geométrico, sino respecto de un punto ecuante distinto de aquel. Más tarde, en el Renacimiento, Copérnico objetará la introducción del ecuante y sustentará su objeción en un criterio estético, como ha sido explicado por Gingerich (1975). Los historiadores de la ciencia saben que el inicio (o shift) de la revolución copernicana reside en la molestia de Copérnico frente a la noción de ecuante, pero no todos los historiadores comprenden bien todo lo que estaba implicado con este «detalle matemático aparentemente trivial», como lo describe Kuhn (1957).

Hasta este punto de nuestra exposición, podemos resumir la trayectoria de la astronomía antigua en tres estadios fundamentales: El modelo de esferas homocéntricas (Eudoxo), el modelo de epiciclo-deferente (Hiparco) y el modelo del ecuante (Ptolomeo). El Almagesto de Ptolomeo es el primer tratado sistemático de astronomía matemática en el cual se desarrolla una explicación cuantitativa, detallada e integral del problema de los planetas. Dentro del estadio ptolemaico, el problema de investigación se podría formular como sigue: ¿Qué combinación particular de deferentes, excéntricas, ecuantes y epiciclos puede explicar los movimientos planetarios con la mayor simplicidad y precisión? Este programa se desarrolló normalmente y no desembocó en una especie de crisis de paradigma, como argumenta Gingerich (1975). Ahora bien, Copérnico vio en la noción de ecuante un recurso inconsistente que atentaba contra el principio de simetría y se puede decir que esta intuición lo condujo a una transformación: la Tierra se convierte en un planeta más y sale del centro del universo.

¿Por qué introdujo Ptolomeo la noción de ecuante? Él sabía que el axioma platónico de la mecánica celeste establece que los astros se mueven uniformemente sobre círculos perfectos en torno a los centros de esos círculos. Ahora bien, con la noción de ecuante, un astro se mueve alrededor de un círculo, pero su movimiento es uniforme en torno a algún otro punto distinto al centro del círculo sobre el que se mueve.De este modo, el centro del epiciclo de un planeta como Venus se mueve regularmente en torno al punctum aequans y no en torno al centro de su deferente:

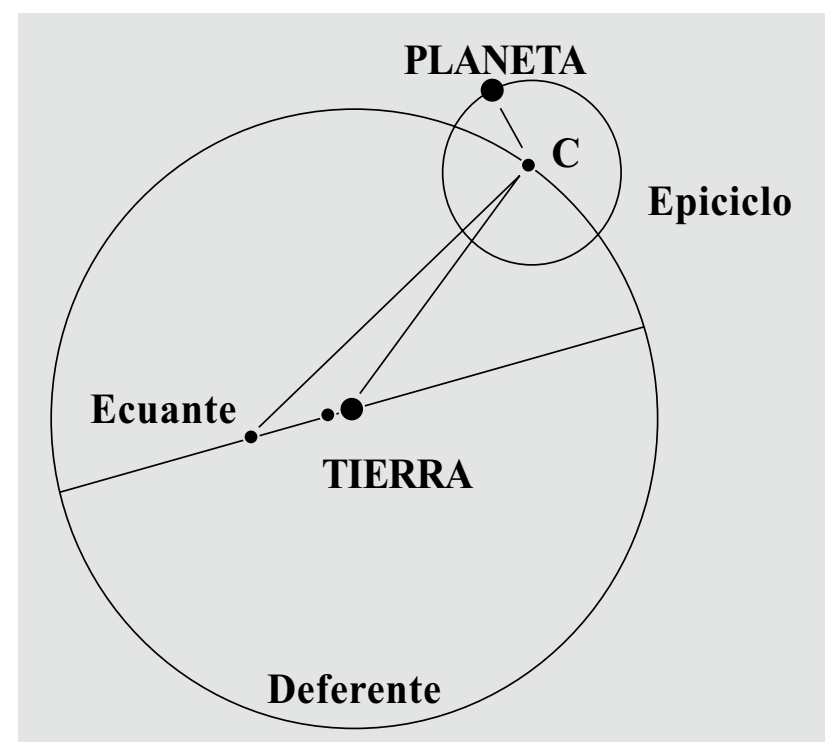


Podría conjeturarse que Ptolomeo propuso el punctum aequans solo como un artilugio matemático de valor instrumental y que no lo conjeturó como un constructo real (Hanson, 1985). Si fuese así, no habría un problema epistemológico sustantivo, dado que como mero mecanismo de cómputo no acarrearía una incompatibilidad con una teoría representativa. La utilidad del ecuante es que logra reconciliar el modelo del epiciclo-deferente con los resultados obtenidos en cuidadosas observaciones. A pesar del valor instrumental del concepto de ecuante, como ya hemos visto, Copérnico lo considera monstruoso y ello lo condujo a su famosa innovación llamada hiperbólicamente revolución copernicana. Copérnico usaba epiciclos menores como los usados por Hiparco, pero ideó un sistema que prescindiera totalmente de los ecuantes, por considerarlos antiestéticos. Apoyado en ese argumento, Copérnico sentenció que el ecuante no era un dispositivo legítimo para ser aplicado a la astronomía. Según él, los efectos del ecuante derruían la armonía simétrica de un cosmos circular y uniforme.

Sobre la base de la exposición precedente, podemos establecer que la llamada revolución copernicana se originó en la presión estética ejercida por el principio de simetría. En efecto, cuando Ptolomeo introduce la noción de ecuante, el axioma platónico de simetría se derrumba y ello determina que en la mente de Copérnico surja la necesidad de restablecer la simetría perdida. Obviamente, Copérnico no tiene una tarea sencilla, dado que, computacionalmente, tiene que igualar o superar el logro de Ptolomeo. Ya no es posible retornar a los modelos iniciales de Eudoxo o de Hiparco. El gran reto de Copérnico es eliminar la noción de ecuante sin perder el triunfo computacional obtenido por Ptolomeo, un modelo superior al de Hiparco. En ese escenario tan peliagudo, Copérnico llega a una genial abducción que se puede explicar en pocas palabras. Se puede restablecer el principio de simetría, arguye Copérnico, sin perder el éxito computacional de Ptolomeo con una innovación: hacer de la Tierra un planeta. En De revolutionibus, Copérnico desarrolla su sistema con un lenguaje técnico, riguroso y matemático, cuya lectura fue una empresa casi imposible, de acuerdo con los resultados de la investigación de Gingerich (2004). En cambio, en el Commentariolus se expone, en términos informales e intuitivos, las razones de su innovación (el texto del Commentariolus se reproduce en Rosen, 1959):

\footnotetext{
"Las teorías planetarias propuestas por Ptolomeo $y$ casi todos los demás astrónomos, aunque
}

guardaban un perfecto acuerdo con los datos numéricos, parecian comportar una dificultad no menor. Efectivamente, tales teorías solo resultaban satisfactorias al precio de tener asimismo que imaginar ciertos ecuantes, en razón de los cuales el planeta parece moverse con una velocidad siempre uniforme, pero no con respecto a su deferente ni tampoco con respecto a su propio centro. Por ese motivo, una teoría de estas caracteristicas no parecía ni suficientemente elaborada ni tan siquiera suficientemente acorde con la razón.

Habiendo reparado en todos estos defectos, me preguntaba a menudo si sería posible hallar un sistema de círculos más racional, mediante el cual se pudiese dar cuenta de toda irregularidad aparente sin tener para ello que postular movimiento alguno distinto del uniforme alrededor de los centros correspondientes, tal y como el principio del movimiento perfecto exige. Tras abordar este problema tan extraordinariamente dificil y casi insoluble, por fin se me ocurrió cómo se podría resolver por recurso a construcciones mucho más sencillas y adecuadas que las tradicionalmente utilizadas, a condición únicamente de que se me concedan algunos postulados".

Así, se puede ver que la noción de ecuante es intolerable para Copérnico porque «no es suficientemente acorde con la razón», esto es, resulta incompatible con el principio de simetría o principio del movimiento perfecto, según la tradición inaugurada por el Timeo. Proponer un modelo que respete el principio de simetría sin perder adecuación computacional, según palabras de Copérnico, viene a ser un problema «casi insoluble». Felizmente, llega a una solución satisfactoria con la condición de aceptar estos siete postulados (Rosen, 1959):

\section{PRIMER POSTULADO}

No existe un centro único de todos los círculos o esferas celestes.

\section{SEGUNDO POSTULADO}

El centro de la Tierra no es el centro del mundo, sino tan solo el centro de gravedad y el centro de la esfera lunar.

\section{TERCER POSTULADO}

Todas las esferas giran en torno al Sol, que se encuentra en medio de todas ellas, razón por la cual el centro del mundo está situado en las proximidades del Sol. 


\section{CUARTO POSTULADO}

La razón entre la distancia del Sol a la Tierra y la distancia a la que está situada la esfera de las estrellas fijas es mucho menor que la razón entre el radio de la Tierra y la distancia que separa a nuestro planeta del Sol, hasta el punto de que esta última resulta imperceptible en comparación con la altura del firmamento.

\section{QUINTO POSTULADO}

Cualquier movimiento que parezca acontecer en la esfera de las estrellas fijas no se debe en realidad a ningún movimiento de esta, sino más bien al movimiento de la Tierra. Así, pues, la Tierra -junto a los elementos circundantes- lleva a cabo diariamente una revolución completa alrededor de sus polos fijos, mientras que la esfera de las estrellas y último cielo permanece inmóvil.

\section{SEXTO POSTULADO}

Los movimientos de que aparentemente está dotado el Sol no se deben en realidad a él, sino al movimiento de la Tierra y de nuestra propia esfera, con la cual giramos en torno al Sol exactamente igual que los demás planetas. La Tierra tiene, pues, más de un movimiento.

\section{SÉPTIMO POSTULADO}

Los movimientos aparentemente retrógrados y directos de los planetas no se deben en realidad a su propio movimiento, sino al de la Tierra. Por consiguiente, este por sí solo basta para explicar muchas de las aparentes irregularidades que en el cielo se observan.

Vemos, pues, que la innovación copernicana se sustenta en la plena adopción del principio de simetría propugnado por Platón en el Timeo. Para descartar el empleo de los ecuantes, Copérnico llega a la abducción que consiste en sacar a la Tierra del centro del universo y dotar de tres movimientos al planeta terrestre. Cabe recordar que la idea del movimiento de la Tierra no era un expediente conceptual inédito que solo se le ocurrió a Copérnico. El mismo señala que no es una idea descabellada dentro de la tradición pitagórica (por ejemplo, el pitagórico Filolao formula una conjetura parecida). Los movimientos de la Tierra tienen la virtud de salvar las irregularidades en el movimiento de los planetas, razón por la cual tienen la bondad heurística de restablecer la simetría abandonada por Ptolomeo. Ahora bien, en la medida en que el principio de simetría es la piedra angular de la astronomía antigua, no hay posibilidad de interpretar que la innovación copernicana fue, en sí misma, revolucionaria. Según nuestra interpretación, la innovación copernicana busca defender acendradamente la tradición antigua de la astronomía y no persigue producir una revolución en la concepción astronómica.

Sin embargo, se habla y se ha hablado mucho de una revolución copernicana, lo que ha significado que la mayoría de historiadores, incluso los más filosóficos, solo traten de comprender la revolución copernicana sin demostrar que tuvo lugar. Esta petitio principii revela hasta qué punto una tradición historiográfica puede ser inexpugnable. Así, por ejemplo, el brillante epistemólogo Imre Lakatos no pone en cuestión la existencia de una revolución copernicana y, más bien, trata de argumentar cómo se ha dado en términos de su propuesta metodológica (Lakatos y Zahar, 1976).

Lakatos propone que la revolución copernicana, incluso, es la verdadera piedra de toque de las distintas filosofías de la ciencia. Define 'revolución copernicana' como la hipótesis de que la Tierra se mueve alrededor del Sol en lugar de suceder al revés. En su trabajo, Lakatos discute la interpretación de la revolución copernicana hecha por el empirismo (inductivistas, probabilistas o falsacionistas), el simplicismo y por autores como Feyerabend. Luego de establecer plausiblemente que las filosofías de la ciencia son esquemas poco promisorios, Lakatos formula su propia interpretación basada en la metodología de los programas de investigación científica.

Tanto el sistema ptolemaico como el sistema copernicano son programas de investigación que entran en rivalidad en la época del Renacimiento. Como programa de investigación, tienen un núcleo firme de ideas medulares muy persistentes y un cinturón protector de hipótesis auxiliares que se exponen a la posible refutación del modus tollens. Para que

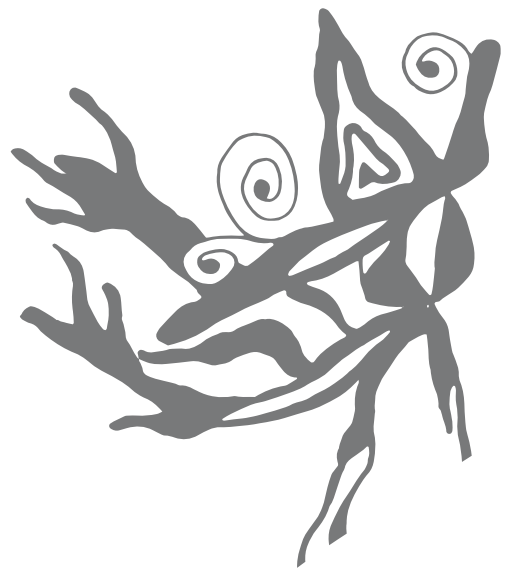
un programa demuestre ser mejor que otro programa, Lakatos propone el triple criterio del progreso científico. De acuerdo con este triple criterio, los programas deben evidenciar progreso teórico (es decir, nuevas e inesperadas predicciones), progreso empírico (es decir, la corroboración de algunas predicciones) y progreso heurístico (es decir, avanzar sin recurrir a hipótesis ad hoc). El análisis de Lakatos muestra claramente que 
el programa copernicano era mejor, desde el punto de vista científico, que el programa de Ptolomeo, pero no demuestra el carácter revolucionario. El mismo Lakatos reconoce que el programa copernicano restaura la heurística tradicional de Platón (lo que denominamos el principio de simetría). Un historiador como Neugebauer (1968) llega, incluso, a decir que el sistema copernicano está plenamente acomodado a la tradición de la astronomía antigua. En conclusión, si bien es cierto que el sistema copernicano es mejor que el sistema ptolemaico, no se puede decir que Copérnico haya inaugurado una nueva era en la astronomía. La obra copernicana debe entenderse como un modo de defender el principio platónico de simetría. ¿Copérnico fue el último de los antiguos o el primero de los modernos? Nos inclinamos a apoyar el primer factor de la disyuntiva.

\section{Referencias bibliográficas}

ÁLVAREZ VITA, E. (2011). «La belleza como guía de la ciencia». Tradición, XI. Diciembre.

BURTT, E. A. (1959). The Metaphysical Foundations of Modern Science. London: Routledge and Kegan Paul.

Casas Navarro, R. y C. Matta (2006). El método científico. Lima: Mantaro.

COPÉRNICO, N. (2009). Sobre las revoluciones (de los orbes celestes). Madrid: Tecnos.

GINGERICH, O. (1975). “"Crisis" versus aesthetic in the Copernican Revolution». Vistas in Astronomy, 17 (1): pp. 85-95.
GINGERICH, O. (2004). The Book Nobody Read. Chasing the revolutions of Nicolaus Copernicus. New York: Walker.

GINGERICH, O. \& J. MACLACHLAN (2005). Nicolaus Copernicus: Making the Earth a Planet. New York: Oxford University Press.

HANSON, N. R. (1985). Constelaciones y conjeturas. Madrid: Alianza.

KUHN, T. (1957). The Copernican Revolution. New York: Vintage Books.

KUHN, T. (1971). La estructura de las revoluciones cientificas. México: FCE.

LAKATOS, I. \& E. ZAHAR (1976). «Why Did Copernicu’s Programme Supersede Ptolemy's?». En R. Westman (ed.). The Copernican Achievement, pp. 354-383. Los Angeles: University of California Press.

NEUGEBAUER, O. (1968). «On the Planetary Theory of Copernicus». Vistas en Astronomy, 10, pp. 89-103.

Platón (1993). Diálogos. Madrid: Tecnos.

ROSEN, E. (1959). Three Copernican Treatises. New York: Dover.

ROSEN, J. (1975). Symmetry discovered. Cambridge: Cambridge University Press.

TATARKIEWCIZ, W. (2001). Historia de seis ideas. Madrid: Tecnos.

WEYL, H. (1952). Symmetry. Princeton: Princeton University Press.

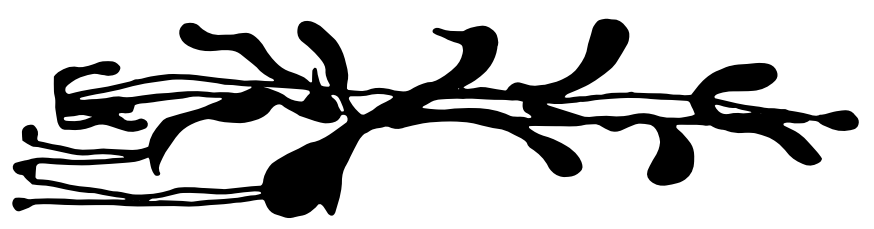

